\title{
Pig carcass tail lesions: the influence of record keeping through an advisory service and the relationship with farm performance parameters
}

\author{
N. van Staaveren ${ }^{1,2 \dagger}$, D. L. Teixeira ${ }^{1 a}$, A. Hanlon ${ }^{2}$ and L. A. Boyle ${ }^{1}$ \\ ${ }^{1}$ Pig Development Department, Teagasc Animal and Grassland Research and Innovation Centre, Moorepark, Fermoy, Co. Cork, P61 C996 Ireland; ${ }^{2}$ School of \\ Veterinary Medicine, University College Dublin, Belfield, Dublin, D04 W6F6 Ireland
}

(Received 4 December 2015; Accepted 20 May 2016; First published online 16 June 2016)

\begin{abstract}
Tail lesions are important pig welfare indicators that could be recorded during meat inspection as they are more visible on the carcass than on the live animal. Tail biting is associated with reduced performance in the bitten pig, but it is not clear whether problems with tail biting are reflected in general farm performance figures. Farm advisory services aim to improve farm productivity which could be associated with improvements in pig welfare. Record keeping forms an integral part of such advisory services. The aim of this study was to examine the influence of record keeping in the Teagasc eProfit Monitor (ePM herds) on the prevalence of tail lesion severity scores in Irish slaughter pigs. In addition, we investigated associations between the prevalence of tail lesion scores and production parameters at farm level in ePM herds. Pigs were observed after scalding/dehairing and tail lesion score (0 to 4), sex and farm identification were recorded. Tail lesion scores were collapsed into none/mild lesions (score $\leqslant 1$ ), moderate lesions (score 2) and severe lesions (score $\geqslant 3$ ). The effect of record keeping (ePM herd) on the different tail lesion outcomes was analysed at batch level using the events/trials structure in generalized linear mixed models (PROC GLIMMIX). Spearman's rank correlations were calculated between average tail lesion score of a batch and production parameters. A total of 13133 pigs were assessed from 73 batches coming from 61 farms. In all, 23 farms were identified as ePM herds. The average prevalence of moderate tail lesions was $26.8 \%$ and of severe tail lesions was $3.4 \%$ in a batch. Batches coming from ePM herds had a lower prevalence of moderate tail lesions than non-ePM herds $(\mathrm{P}<0.001)$. Average tail lesion score was negatively associated with age $(\mathrm{P}<0.05)$ and weight $(\mathrm{P}<0.05)$ at sale/transfer of weaners, and tended to be positively associated with the number of finishing days $(\mathrm{P}=0.06)$. In addition, the prevalence of severe tail lesions was negatively associated with average daily gain in weaners $(\mathrm{P}<0.05)$ and tended to do so with average daily gain in finishers $(\mathrm{P}=0.08)$. This study provides the first indication that record keeping through an advisory service may help to lower the risk of tail biting, which is associated with improved farm performance.
\end{abstract}

Keywords: tail lesions, meat inspection, pigs, record keeping, farm performance

\section{Implications}

Pig advisory services aim to improve farm productivity through better housing, management and nutrition and could therefore have a positive effect on pig welfare. Detailed record keeping of financial and farm performance provides an evidence base to target (and appraise) advice. This research showed that farms which use the record keeping aspect of an advisory service had fewer moderate tail lesions as observed on the carcass than farms that did not keep records. Furthermore, tail lesion score was associated

\footnotetext{
a Present address: Departamento de Ciencias Animales, Facultad de Agronomía e Ingeniería Forestal, Pontificia Universidad Católica de Chile, Chile.

${ }^{\dagger}$ E-mail: nienke.vanstaaveren@teagasc.ie
}

with farm performance characteristics indicating a relationship between tail biting and record keeping through an advisory service. Recording tail lesions at meat inspection could aid in monitoring the problem and help advisory services in informing general herd health and welfare management plans.

\section{Introduction}

Tail lesions are pig welfare indicators with good potential to record during meat inspection (Harley et al., 2012a and 2014). Tail lesions are an indication of tail biting behaviour that reflects impaired welfare often related to disharmony between the pig and its environment (Smulders et al., 2006). 
Bracke et al. (2013) conducted a survey of 520 pig farmers and concluded that biting of tails, ears and limbs is a major welfare problem in pig production. Harley et al. performed two studies of slaughter pigs in abattoirs in the Republic of Ireland and Northern Ireland and found that $60 \%$ to $70 \%$ of the observed slaughter pigs had detectable tail lesions on the carcass (Harley et al., 2012b and 2014). Tail lesions are more visible on the carcass, especially less severe bite marks, compared with the live animal suggesting that recording tail lesions during meat inspection is more accurate (Velarde et al., 2005; Harley et al., 2012a; Carroll et al., 2015; van Staaveren et al., 2015). An undocked, uninjured tail at slaughter is considered a gold standard for pig welfare and suggested as an 'iceberg indicator' capable of providing an overall picture of the health/ welfare of pigs (Farm Animal Welfare Council (FAWC), 2009; European Food Safety Authority (EFSA), 2012).

Tail biting is associated with reduced performance in pigs. Sinisalo et al. (2012) reported that tail-bitten pigs had $1 \%$ to $3 \%$ reduction in average daily gain when adjusted for genetic merit. Similar results were found by Zonderland et al. (2010) with a decreased feed intake and daily weight gain, though differences were found between sex and group compositions. The increased risk of pathological lesions, carcass trimmings/condemnations and reduced carcass weight in pigs with even moderate tail lesions (Walker and Bilkei, 2006; Kritas and Morrison, 2007; Harley et al., 2014; Teixeira et al., 2016; van Staaveren et al., 2016) shows the importance of recording both moderate and severe tail damage at meat inspection (EFSA, 2007). It is unclear if the relationship between tail biting and reduced productivity of the affected individual is also found at farm level, that is, if farms with higher levels of tail biting have poorer performance figures, although it is reasonable to suspect that this would be the case. Tail biting is a sign that the animal cannot cope within its environment and can increase restlessness in the pen (EFSA, 2007). This could possibly influence the performance of all the pigs on a farm, even those which do not show any signs of having been bitten.

Farmers have an economic framing of animal welfare and place most emphasis on good health as determined by production numbers, growth and absence of diseases (Benard et al., 2014). In this regard, tail biting is an important welfare problem because of its negative effects on production and health (EFSA, 2007). Farm-specific advice and financial incentives can help farmers to reduce the risk of tail biting, however this depends on whether the advice is practical, has other negative consequences or is too expensive to implement (Taylor et al., 2012). Advisory services can avoid these pitfalls, because they are aware of practical issues on the farms, and are trusted by their clients (Benard et al., 2014). Advisory services aim to improve farm productivity through better housing, management and nutrition of pigs. Detailed record keeping of financial and farm performance figures is fundamental to this (McCutcheon and Glover, 2014). Krug et al. (2015) hypothesized that dairy farms with poor welfare were less likely to keep comprehensive records. It is not known if there is also a beneficial effect of record keeping on pig welfare. Furthermore, the extent to which the beneficial effect of record keeping on farm productivity and potentially animal welfare is due to the record itself or the type of farmer (e.g. farming styles, perspectives) is not known. For example, Verstegen and Huirne (2001) reported that while some producers see record keeping as 'essential for future planning' others may view it as a 'necessary chore'. It is conceivable that the latter group would not only be less likely to keep good records but could also be less motivated in general regarding farm improvements. It may be that only proactive, progressive farmers choose to undertake record keeping in the first place and that this is equally important in 'driving' the associated improvements seen at farm level (Doye et al., 2000).

The aim of this study was to investigate the prevalence of tail lesion scores observed on the carcass and to examine the influence of record keeping through an advisory service on this prevalence in Irish slaughter pigs. In addition, we investigated associations between the prevalence of tail lesions and production parameters in those farms which kept comprehensive records.

\section{Material and methods}

\section{Abattoir visits}

Visits were carried out at two abattoirs (A, B) in the Republic of Ireland during June and July 2014. In order to obtain an equal number of pigs and herds inspected from each abattoir, data collection occurred over 4 consecutive days in abattoir A (10000 pigs/week; 4 pigs/min) and 3 consecutive days in abattoir B (10500 pigs/week; 6 pigs/min). During these visits, two trained observers recorded tail lesion scores for all pigs on the line during alternate $1.5 \mathrm{~h}$ periods. Pigs were observed on the slaughter line after dehairing and scalding and the herd number, sex and tail lesion score was recorded for each pig.

\section{Tail lesions}

Tail lesions were scored according to severity using a 0 to 5 scale (Table 1) (adapted from Kritas and Morrison, 2007; Harley et al., 2012b).

\section{eProfit Monitor status and farm performance}

The Teagasc advisory service provides farm consultancy visits, herd performance and financial monitoring, organizes discussion groups and provides education and training for the pig sector. All Irish pig producers can avail of these services under the Teagasc/lrish Farmers Association Pig Joint Programme (Carroll, 2013). However, there is considerable variation between producers regarding the extent to which they avail of these services (McCutcheon and Glover, 2014). A key component of herd performance and financial monitoring is record keeping using the Teagasc eProfit Monitor (ePM), which records over 150 parameters detailing technical and financial performance data of the herd. Herd numbers were used to identify clients which avail of the ePM 
van Staaveren, Teixeira, Hanlon and Boyle

Table 1 Tail lesion scoring system for pig carcasses (adapted from Kritas and Morrison, 2007; Harley et al., 2012b)

\begin{tabular}{ll}
\hline \hline Score & Description \\
\hline 0 & No evidence of tail biting \\
1 & Healed or mild lesions \\
2 & Evidence of chewing or puncture wounds, but no \\
& evidence of swelling \\
3 & Evidence of chewing or puncture wounds with \\
& swelling and signs of possible infection \\
4 & Partial loss of the tail \\
5 & Total loss of the tail \\
\hline \hline
\end{tabular}

service (hereafter 'ePM herds'). A total of 23 herds (38\%) were identified as ePM herds and 38 as non-ePM herds (the latter group may have included clients with varying degrees of contact with the Teagasc pig advisory service as well as non-clients). Performance data in the ePM are recorded over a 13-week period and for this study we collected data from 6 months before the abattoir observations (e.g. January to March, April to June 2014).

\section{Statistical analysis}

Interobserver reliability was determined by scoring tail lesions in pigs on a day before the trial started. Levels of agreement were calculated based on the percentage of pigs which were assigned the same score by both observers. A quadratic weighted $\kappa$ was calculated to account for agreements by chance while giving reduced partial credit to disagreements as they are more distant from each other. A $\kappa$ between 0.8 and 1.0 indicates very good to excellent agreement.

All statistical procedures were conducted using SAS V9.3 (SAS Institute Inc., Cary, NC, USA). Tail lesion scores were collapsed into none/mild lesions (score $\leqslant 1$ ), moderate lesions (score 2) and severe lesions (score $\geqslant 3$ ). Data from batches of $<10$ pigs were omitted from the analysis. The prevalence of the different tail lesion outcomes were calculated using descriptive statistics for each batch. Data were analysed at batch level. Residuals were checked for normality and transformed where necessary. The effect of record keeping (ePM herd) on the different tail lesion outcomes was analysed using the events/trials structure in generalized linear mixed models (PROC GLIMMIX). Abattoir was not included in the model as this was partially confounded with ePM herds (i.e. more batches observed in abattoir B came from ePM herds).

Out of the 23 ePM herds, 21 (91\%) of the herd owners/ managers gave permission to access their ePM data. Farm performance data of seven herds were omitted from the analysis due to missing data (four) and restocking of the herd (three) during the relevant time period. This led to a final group of 14 herds (61\% of ePM herds) with useable farm performance data collected from the ePM system. The production parameters of interest are described in Table 2.

A mean tail lesion score was calculated for each herd. Spearman's rank correlations were calculated between tail lesion prevalence (average tail lesion score, prevalence of moderate or severe tail lesions) and production parameters. $\alpha$ Level for determination of significance was 0.05 and tendencies are reported from 0.05 and 0.10 .

\section{Results}

\section{Descriptive results}

A level of agreement of $71 \%$ was reached between observers for tail lesion scores based on 353 pigs scored during the interobserver reliability testing. The quadratic $\kappa$ was calculated at 0.8 between the two observers showing good interobserver reliability.

Data from 13155 pigs were collected across the two abattoirs. A final study population of 13133 was obtained after removing pigs from batches $<10$. A total of 30 batches from 27 farms were observed in abattoir A (5829 pigs) and 38 batches from 35 farms were observed in abattoir B (7304 pigs). Batch size ranged from 20 to 482 pigs. One farm sent pigs to both abattoirs, meaning that in total 73 batches from 61 different farms were sampled. Only $20 \%$ of the farms that were observed in abattoir A were identified as ePM herds (six), while about $50 \%$ of the farms supplying abattoir $B$ were ePM herds (18).

\section{Tail lesions}

Moderate and severe tail lesions were present in $25.2 \%$ and $3.1 \%$ of the pigs, respectively. Batch-level prevalence ranged from $3.2 \%$ to $70 \%$ for moderate and $0 \%$ to $21.4 \%$ for severe tail lesions (Figure 1). Moderate tail lesions were found in all batches, while severe tail lesions were observed in 56 of the 73 batches. On average, $26.8 \%$ of pigs in a batch were affected by moderate tail lesions, however in $47.9 \%$ of the batches this figure was higher. For severe tail lesions, the average batch-level prevalence was 3.4\% but higher percentages of pigs with severe tail lesions were found in $28.8 \%$ of the batches.

\section{Tail lesions and eProfit Monitor status}

Average batch size tended to be larger in ePM herds $(216.6 \pm 18.6)$ than non-ePM herds $(176.9 \pm 14.2$, $P=0.09)$. The 23 herds that kept records in the ePM supplied $27(37 \%)$ of the observed batches and accounted for almost $40 \%$ of all observed pigs. These ePM batches had a higher percentage of pigs with none/mild tail lesions (Table 3, $P<0.001$ ) and a lower percentage of pigs with moderate tail lesions $(P<0.001)$ than non-ePM batches. No difference between ePM and non-ePM batches was found for the prevalence of severe tail lesions $(P>0.05)$.

\section{Tail lesions and production parameters}

Reliable production data were obtained for a final 14 herds (17 batches) and associations between tail lesions and production parameters could only be determined for these herds. Three herds sent two batches during the study period and therefore the average of the two batches were used for 
Pig tail lesions in relation to record keeping

Table 2 Description of production parameters collected from the eProfit Monitor records

\begin{tabular}{llcc}
\hline \hline Category & Production parameter & Mean \pm SE & Range (minimum to maximum) \\
\hline \multirow{2}{*}{ Stocks and balances } & Average herd size & $928.1 \pm 197.48$ & 138.0 to 2288.0 \\
& Litter/sow per year & $2.3 \pm 0.02$ & 2.18 to 2.54 \\
& Farrowing rate (\%) & 71.9 to 97 \\
& Born alive per litter & $83.1 \pm 3.76$ & 11.5 to 14.1 \\
& Weaner mortality (\%) & $13.1 \pm 0.20$ & 1.2 to 6.1 \\
& Finisher mortality (\%) & $2.6 \pm 0.34$ & 1.2 to 4.8 \\
& No. pig produced/sow per year $(\mathrm{kg})$ & $2.3 \pm 0.29$ & 23.2 to 29.1 \\
Weaning to sale & Pig meat produced/sow per year (kg) & $26.1 \pm 0.50$ & 1144.0 to 2325.0 \\
& Age at sale (days) & $1964.1 \pm 77.7$ & 151.0 to 197.0 \\
Weaner & Average daily gain (g) & $174.2 \pm 3.06$ & 598.0 to 810.0 \\
& Feed conversion & $683.6 \pm 14.55$ & 2.2 to 2.6 \\
& Age at sale/transfer (days) & $2.4 \pm 0.03$ & 80.4 to 108.7 \\
Finisher & Weight at sale/transfer (kg) & $95.0 \pm 2.21$ & 29.1 to 45.0 \\
& Average daily gain (g) & $38.3 \pm 1.32$ & 392.0 to 573.0 \\
& Feed conversion & $486.1 \pm 16.79$ & 1.5 to 2.7 \\
Noverall & No. finishing days & $1.8 \pm 0.08$ & 62.3 to 96.9 \\
\hline
\end{tabular}

${ }^{1}$ Data were only recorded for seven herds.

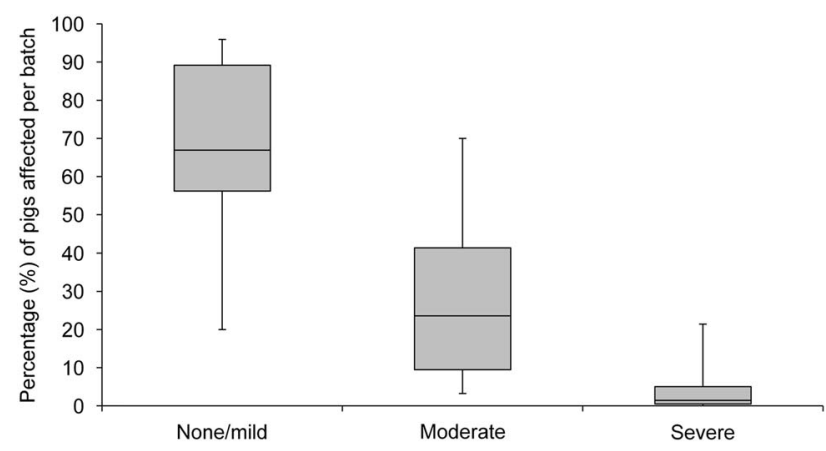

Figure 1 Variation between batches of pigs in tail lesions with different degrees of severity (none/mild: score $\leqslant 1$, moderate: score 2 , severe: score $\geqslant 3$ ).

Table 3 The average percentage of pigs (LS mean \pm SE) in a batch with none/mild (score $\leqslant 1$ ), moderate (score 2 ) and severe (score $\geqslant 3$ ) tail lesions according to eProfit Monitor (ePM) record keeping status

\begin{tabular}{lrrrrrr}
\hline \hline & \multicolumn{2}{c}{ ePM record keeping } & & & \\
\cline { 2 - 3 } & \multicolumn{2}{c}{ Yes } & No & OR $^{1}$ & $95 \% \mathrm{Cl}$ & $P$-value \\
\hline Tail lesions (\%) & & & & & \\
$\quad$ None/mild & $80.1 \pm 0.55$ & $66.2 \pm 0.53$ & 2.1 & 1.89 to 2.24 & $<0.001$ \\
Moderate & $17.0 \pm 0.52$ & $30.6 \pm 0.52$ & 0.5 & 0.43 to 0.51 & $<0.001$ \\
Severe & $2.8 \pm 0.23$ & $3.3 \pm 0.20$ & 0.9 & 0.70 to 1.06 & $>0.05$ \\
\hline
\end{tabular}

${ }^{1}$ Odds ratio reported for batches from farms with record keeping compared with no record keeping in the ePM.

these herds. As ePM herds showed less tail lesions this could have influenced the results and results should be interpreted with caution.
Correlations were found between the average tail lesion score of a herd and several of the herd production indicators (Table 4). A significant negative correlation was found between average tail lesion score and age at sale/transfer $(P<0.05)$ and weight at sale/transfer of weaners $(P<0.05)$. Further investigation revealed that the percentage of pigs with severe tail lesions in a herd was negatively correlated with average daily gain in weaners $(P<0.05)$. The average tail lesion score of a herd tended to be positively correlated with the number of finishing days $(P=0.06)$ and the percentage of pigs with severe tail lesions tended to be negatively correlated with average daily gain in the finishers $(P=0.08)$. Finally, a moderate negative correlation was found between the percentage of pigs with severe tail lesions and the average live weight sold for finishers $(P<0.05$; Table 4$)$.

\section{Discussion}

The aims of this study were to examine the influence of record keeping with an advisory service on the prevalence of tail lesions and to establish relationships between tail lesion scores and farm performance figures. This study confirmed that tail lesions are common in Irish slaughter pigs. Figures for the prevalence of moderate $(25 \%)$ and severe $(3.1 \%$ of pigs affected) tail lesions correspond well with previous work in Irish slaughter pigs (Harley et al., 2014). This illustrates that there is a consistent tail biting problem on Irish pig farms, despite most pigs being tail docked (Harley et al., 2012b). While Irish farmers consider tail biting a problem there is a tendency amongst both pig producers and processors to focus on severe tail bites (EFSA, 2007 and 2014; 
van Staaveren, Teixeira, Hanlon and Boyle

Table 4 Correlations between production parameters $(\mathrm{n}=14$ herds) and average tail lesion score, the prevalence of moderate tail lesions (score 2) and severe tail lesions (score $\geqslant 3$ )

\begin{tabular}{lccc}
\hline \hline Production parameter ${ }^{1}$ & Tail lesion score & Moderate tail lesions (\% of pigs) & Severe tail lesions (\% of pigs) \\
\hline Weaning to sale - average daily gain (g) & $\mathrm{Ns}$ & $\mathrm{Ns}$ & $-0.54^{*}$ \\
Weaner - age at sale/transfer (days) & $-0.54^{*}$ & $-0.50^{\dagger}$ & $\mathrm{Ns}$ \\
Weaner - weight at sale/transfer (kg) & $-0.63^{*}$ & $-0.56^{*}$ & $\mathrm{Ns}$ \\
Finisher - no. finishing days & $0.52^{\dagger}$ & $\mathrm{Ns}$ & $\mathrm{Ns}$ \\
Finisher - average daily gain (g) & $\mathrm{Ns}$ & $\mathrm{Ns}$ & $-0.48^{\dagger}$ \\
Finisher - average live weight sold $(\mathrm{kg})$ & $\mathrm{Ns}$ & $\mathrm{Ns}$ & $-0.61^{*}$ \\
\hline
\end{tabular}

${ }^{1}$ Only productions parameters for which associations were found are reported. Other production parameters as listed in Table 2 were not significant (Ns). ${ }^{*} P<0.05$, ${ }^{\dagger} 0.05 \leqslant P \leqslant 0.1$.

Bracke et al., 2013; Devitt et al., 2016). Furthermore, producers show a tolerance of tail biting behaviour as it is considered to be a sporadic problem caused by factors outside of their control (e.g. season) (Devitt et al., 2016). However, moderate tail lesions which appear to be related to chronic tail manipulation and chewing as opposed to overt biting, could escalate into tail biting outbreaks (Taylor et al., 2010) and should therefore be avoided. The large variation between batches, especially in the prevalence of moderate tail lesions, indicates that on some farms there is less tail-directed behaviour performed by the pigs. However, the multifactorial nature of the tail biting problem makes it difficult to know which specific on-farm factors are responsible for this (EFSA, 2007). Recording of tail lesions at slaughter could help in monitoring tail biting behaviour over time, and possibly overall health and welfare, whereby an undocked, uninjured tail at slaughter is considered the gold standard (FAWC, 2009; EFSA, 2012).

The results of this study indicate that keeping of financialand performance-related records in the ePM system was associated with a lower prevalence of moderate tail lesions. Moinard et al. (2003) found that tail biting is more common on farms which are less well managed. Record keeping is associated with high standards of management (McCutcheon and Glover, 2014; Krug et al., 2015) and could explain the lower prevalence of moderate tail lesions in ePM herds. By monitoring ePM figures, Teagasc advisors are able to assist pig producers in making decisions and evaluating responses to changes in management, feeding or housing. Conceivably, where such changes improve pig performance there are likely to be associated improvements in pig welfare possible reflected in a lower prevalence of tail biting. Furthermore, farmers may be more willing to modify management practices, on the recommendation of an advisor, as there is a basis of trust in the farmer-advisor relationship and advisors are more aware of practical issues (Taylor et al., 2012; Benard et al., 2014). Nevertheless, the impact that different producer perspectives have on their likelihood to keep records and therefore on the improvements associated with record keeping cannot be discounted. Doye et al. (2000) described farmers perceived to have successful information systems as analytic in nature, committed to lifelong learning, and farmers who saw the information system as highly valuable to achieving their goals.
No comparison was made to more typical producers with poorer information systems but it is possible that they are generally less motivated (Doye et al., 2000). Further research is needed to identify differences in farmer perspectives and personalities in terms of motivation to join record keeping schemes.

No other information from non-ePM herds could be obtained. It is likely that ePM and non-ePM herds differed in other underlying aspects which contributed to the differences found in tail lesion prevalence. For example, batch size of ePM herds was larger than non-ePM herds and this could suggest that the lowered risk of moderate tail lesions in ePM herds was mediated to some extent by herd size. However, the relationship between herd size and tail biting is often confounded with other factors such as type of production system, degree of automation and other management factors (EFSA, 2007). Large-scale pig units are often viewed as negative for tail biting owing to their association with higher ratio of animals to stockpersons (Moinard et al., 2003) but could also be positive for pig welfare because of the likelihood of being more intensively managed (e.g. higher degree of automation) (Verstegen and Huirne, 2001).

The associations found between tail lesions observed on the carcass and farm production parameters provide the first indication that tail biting is associated with productivity indicators at a farm level. It is important to remember that the results discussed here are associations and no deductions could be made regarding possible causal relationships. As these data only applied to the ePM herds, which had fewer tail lesions than non-ePM herds, it is possible that different results could be found in a larger sample. Therefore, the associations found here are considered exploratory and results should be interpreted with caution. In addition, the observations of batches of pigs at the abattoir represented 'a point' observation (both in time and in number of pigs) while the performance data reflected a period of 6 months covering all pigs in the farm. It would be interesting to examine if carcass tail lesion prevalence mirror temporal changes in performance data.

It is known that tail biting impedes the production performance of pigs that are bitten as shown in lower feed intake, lower average daily gain and lower carcass weight (Zonderland et al., 2010; Sinisalo et al., 2012; 
Valros et al., 2013; Harley et al., 2014). The average tail lesion score of a herd was associated with age and weight at sale/transfer for weaners. Weight at sale/transfer decreased with increasing average tail lesion score. This is in agreement with Beattie et al. (2005) who found that pigs that spent more time tail biting were lighter at weaning and also tended to be lighter at 7 weeks of age. Thus, weaning of smaller pigs could lead to more challenges in the grower-finisher period and influence the risk of tail biting. A higher prevalence of tail lesions in a batch is also associated with a lower average cold carcass weight (Carroll et al., 2015). Similarly, we found that as the percentage of severe tail lesions increased the average live weight at which finisher pigs were sold decreased. In addition, the number of finishing days tended to increase when the average tail lesion score of the herd increased. This could reflect the fact that tail-bitten pigs grow slower (Zonderland et al., 2010; Sinisalo et al., 2012) and is further supported by our findings that severe tail lesions were correlated with a lower average daily gain.

\section{Conclusion}

This study provides the first indication that record keeping with an advisory service is associated with a lower risk of tail lesions recorded on the carcass and that such lesions are associated with characteristics of general farm productivity. Further research is needed to identify differences in farmers' motivation to keep records, perceptions on tail biting and presence of risk factors for tail biting on farms that are taking part in the advisory services or not. It may be that in informing general herd health and welfare management plans the advisory services are helping to reduce the risk of tail biting. This role could be aided by recording information on moderate and severe tail lesions at meat inspection.

\section{Acknowledgements}

The authors would like to extend their thanks to the managers and personnel of the abattoirs for kindly allowing them to perform this experiment. Their gratitude is also expressed towards the pig farmers for allowing them to access their herd performance data. In addition, the authors would like to thank the Teagasc pig advisory staff for their assistance in farmer recruitment and collecting the data. They would like to acknowledge Prof Simon More and Dr Edgar Garcia Manzanilla for their insights on the statistical analysis. A special thanks to Nora Roussel for her enthusiasm and help with the data collection. This study was part of the PIGWELFIND project funded by the Research Stimulus Fund (11/S/107) of the Irish Department of Agriculture, Food and Marine under the National Development Plan (2007 to 2013).

\section{References}

Beattie VE, Breuer K, O'Connell NE, Sneddon IA, Mercer JT, Rance KA, Sutcliffe MEM and Edwards SA 2005. Factors identifying pigs predisposed to tail biting. Animal Science 80, 307-312.
Benard M, Schuitmaker TJ and de Cock Buning T 2014. Scientists and Dutch pig farmers in dialogue about tail biting: unravelling the mechanism of multi-stakeholder learning. Journal of Agricultural and Environmental Ethics 27, 431-452.

Bracke MBM, de Lauwere CC, Wind SMM and Zonderland JJ 2013. Attitudes of Dutch pig farmers towards tail biting and tail docking. Journal of Agricultural and Environmental Ethics 26, 847-868.

Carroll C 2013. Joint programme update. Pig Farmers' Conference 2013 Cavan Crystal Hotel, 22 October and Horse \& Jockey Hotel, 23 October 2013, Ireland.

Carroll GA, Boyle LA, Teixeira DL, van Staaveren N, Hanlon A and O'Connell NE 2015. Effects of scalding and dehairing of pig carcasses at abattoirs on the visibility of welfare-related lesions. Animal 10, 460-467.

Devitt C, Boyle L, Teixeira DL, O'Connell NE, Hawe M and Hanlon A 2016. Pig producer perspectives on the use of meat inspection as an animal health and welfare diagnostic tool in the Republic of Ireland and Northern Ireland. Irish Veterinary Journal 69, 1-9.

Doye D, Jolly R, Hornbaker R, Cross T, King RP, Lazarus WF and Yeboah A 2000. Case studies of farmers' use of information systems. Review of Agricultural Economics 22, 566-585.

European Food Safety Authority (EFSA) 2007. Scientific report on the risks associated with tail biting in pigs and possible means to reduce the need for tail docking considering the different housing and husbandry systems (Question No EFSA-Q-2006-013). The EFSA Journal (Annex) 611, 1-13.

EFSA 2012. Scientific opinion on the use of animal-based measures to assess welfare in pigs. The EFSA Journal 10, 2512.

EFSA 2014. Scientific opinion concerning a multifactorial approach on the use of animal and non-animal-based measures to assess the welfare of pigs. The EFSA Journal 12, 3702.

Farm Animal Welfare Council (FAWC) 2009. Farm animal welfare in Great Britain: past, present and future. FAWC, London, UK.

Harley S, Boyle L, O'Connell N, More S, Teixeira D and Hanlon A 2014. Docking the value of pigmeat? Prevalence and financial implications of welfare lesions in Irish slaughter pigs. Animal Welfare 23, 275-285.

Harley S, More S, Boyle L, O'Connell N and Hanlon A 2012a. Good animal welfare makes economic sense: potential of pig abattoir meat inspection as a welfare surveillance tool. Irish Veterinary Journal 65, 11-22.

Harley S, More SJ, O'Connell NE, Hanlon A, Teixeira D and Boyle L 2012b. Evaluating the prevalence of tail biting and carcase condemnations in slaughter pigs in the Republic and Northern Ireland, and the potential of abattoir meat inspection as a welfare surveillance tool. Veterinary Record 171, 621-627.

Kritas SK and Morrison RB 2007. Relationships between tail biting in pigs and disease lesions and condemnations at slaughter. Veterinary Record 160, 149-152.

Krug C, Haskell MJ, Nunes T and Stilwell G 2015. Creating a model to detect dairy cattle farms with poor welfare using a national database. Preventive Veterinary Medicine 122, 280-286.

McCutcheon G and Glover K 2014. Live records for benchmarking Teagasc ePM. Pig Farmers' Conference 2014, Horse \& Jockey Hotel, 21 October and Cavan Crystal Hotel, 22 October 2014, Ireland.

Moinard C, Mendl M, Nicol CJ and Green LE 2003. A case control study of on-farm risk factors for tail biting in pigs. Applied Animal Behaviour Science 81, 333-355.

Sinisalo A, Niemi JK, Heinonen M and Valros A 2012. Tail biting and production performance in fattening pigs. Livestock Science 143, 220-225.

Smulders D, Verbeke $G$, Mormède $P$ and Geers R 2006. Validation of a behavioral observation tool to assess pig welfare. Physiology \& Behavior 89, 438-447.

Taylor NR, Main DCJ, Mendl M and Edwards SA 2010. Tail-biting: a new perspective. The Veterinary Journal 186, 137-147.

Taylor NR, Parker RMA, Mendl M, Edwards SA and Main DCJ 2012. Prevalence of risk factors for tail biting on commercial farms and intervention strategies. The Veterinary Journal 194, 77-83.

Teixeira D, Harley S, Hanlon A, O'Connell NE, More S, Manzanilla E and Boyle L 2016. Study on the association between tail lesion score, cold carcass weight and viscera condemnations in slaughter pigs. Frontiers in Veterinary Science 3, 10.3389/fvets.2016.00024. 
Valros A, Munsterhjelm C, Puolanne E, Ruusunen M, Heinonen M, Peltoniemi OAT and Poso AR 2013. Physiological indicators of stress and meat and carcass characteristics in tail bitten slaughter pigs. Acta Veterinaria Scandinavica 55, 75-75.

van Staaveren N, Teixeira DL, Hanlon A and Boyle LA 2015. The effect of mixing entire male pigs prior to transport to slaughter on behaviour, welfare and carcass lesions. PLoS One 10, e0122841.

van Staaveren N, Vale AP, Manzanilla EG, Teixeira DL, Leonard FC, Hanlon A and Boyle LA 2016. Relationship between tail lesions and lung health in slaughter pigs. Preventive Veterinary Medicine 127, 21-26.

Velarde A, Dalmau A, Fàbrega E and Manteca X 2005. Health and welfare management of pigs based on slaughterline records. The 56th Annual Meeting of the European Association for Animal Production, 2-5 June 2015, Uppsala, Sweden.

Verstegen JAAM and Huirne RBM 2001. The impact of farm management on value of management information systems. Computers and Electronics in Agriculture 30, 51-69.

Walker PK and Bilkei G 2006. Tail-biting in outdoor pig production. The Veterinary Journal 171, 367-369.

Zonderland JJ, Bracke MBM, den Hartog LA, Kemp B and Spoolder HAM 2010. Gender effects on tail damage development in single- or mixed-sex groups of weaned piglets. Livestock Science 129, 151-158. 